\title{
Las oposiciones para las bibliotecas y archivos
}

Debate coordinado por CARLOS MIGUEL TEJADA ARTIGAS

Profesor titular de la Facultad de Ciencias de la Documentación de la Universidad Complutense de Madrid

Orcid: 0000-0002-2767-5636

\begin{abstract}
Resumen
A partir de seis preguntas, se realiza un debate sobre las oposiciones a los cuerpos y escalas de bibliotecas y archivos. Las temáticas que se abordan son: la evolución de las convocatorias de las oposiciones, la pertinencia de los ejercicios y temarios y la formación de los opositores. También se dialoga sobre dos problemas que se dan en los niveles superiores sobre todo de bibliotecas: el número escaso de candidatos que se presentan y el hecho que muchas de las plazas ofertadas se queden desiertas. Además del moderador, intervienen cinco profesionales que conocen muy de cerca estos procesos y también se recoge a través de una persona el punto de vista del opositor.
\end{abstract}

Palabras clave

Oposiciones; bibliotecas; archivos; temarios

\begin{abstract}
From six questions, we present a debate on the public examination to the bodies and scales of libraries and archives. The topics addressed are: the evolution of the public calls, the pertinence of the exercises and collections of themes and the training of candidates. There is also a dialogue about two problems that occur at the upper levels, especially in libraries: the small number of candidates applying to the public calls and the fact that many of the positions offered are not covered due to a lack of candidates passing the selection process. In addition to the moderator, five professionals who know these processes very closely participate in the debate and the point of view of the the candidates is also reflected by the inclusion of the contributions of one of them.
\end{abstract}

Keywords

Public examination; libraries; archives; collections of themes

\section{Introducción}

En la próxima década se va a producir un proceso de jubilación masiva de los empleados públicos. Ejemplo de ello es el caso de la Administración General del Estado, donde en los próximos diez años se jubilará más del $50 \%$ de su personal y tan solo el $12 \%$ tiene menos de 40 años (Ministerio de Política Territorial y Función Pública, 2021).

Bajo el principio de que el acceso abierto a los resultados de investigación acelera el avance del conocimiento, todos los contenidos de la edición electrónica de CLIP se distribuyen bajo una licencia de uso y distribución Creative Commons Reconocimiento-NoComercialCompartirlgual 3.0 España (CC BY-NC-SA 3.0 ES). 
Entre este personal de las Administraciones Públicas se encuentran los bibliotecarios y archiveros. Así, en los últimos años las plazas convocadas en nuestra área son numerosas. A modo de ejemplo en este 2021, el Ministerio de Cultura y Deporte ha convocado 22 plazas libres de facultativos de bibliotecas y 46 de archivos, y 84 plazas de ayudantes de bibliotecas y 74 de archivos.

Es pues, fundamental, analizar la selección de personal y el procedimiento establecido a tal efecto. Este es un proceso de toma de decisión sobre la adecuación de los candidatos a los puestos ofertados. Políticos y medios de comunicación ya han planteado la idoneidad del sistema actual de oposiciones, en el que prima la memorización de contenidos teóricos. Otros procesos de selección, como los de la propia Unión Europea o de países tan importantes de nuestro entorno como Portugal, Francia o Alemania, están más centrados en las capacidades para desempeñar un puesto de trabajo. Además, no hay que olvidar que el Real Decreto 5/2015, de 30 de octubre del Estatuto Básico del Empleado Público establece que las Administraciones Públicas deben de tener entre sus principios la adecuación entre el contenido de los procesos selectivos y las funciones o tareas a desarrollar.

De gran interés e importancia es el documento publicado este año por el Ministerio de Política Territorial y Función Pública: Orientaciones para el cambio en materia de selección en la Administración General del Estado (https://www.mptfp.gob.es/dam/es/portal/funcionpublical secretaria-general-funcion-publica/Actualidad/2021/05/orientacionescambio.pdfo). El objetivo de este documento es sentar las bases para la modificación de los procesos selectivos en la Administración Pública y, aunque los cambios no van a ser inminentes, sí que supone un cambio de tendencia, ya que muestra la conciencia de que "los procesos selectivos actuales parecen haberse quedado superados y dan síntomas de agotamiento". Resalta el hecho de que el modelo vigente se basa sobre todo en acreditar unos conocimientos teóricos, cosa que no está en sintonía con el nuevo modelo de universidad implantado tras el Proceso de Bolonia, en el que prima la adquisición de competencias por parte del alumnado. Se pretende alcanzar un nuevo modelo flexible y adaptable y que aborde gradualmente "la transición desde el predominio en las oposiciones de las pruebas memorísticas a la realización de pruebas que valoren las competencias además de los conocimientos de las personas aspirantes".

Asimismo, presenta una serie de orientaciones de las que se puede destacar tres de ellas:

- Adaptar los procesos selectivos a la diversidad de funciones desempeñadas en el sector público.

- Avanzar hacia un modelo que valore las competencias además de los conocimientos de las personas aspirantes, con un fundamento metodológico sólido anclado en la validez de las pruebas selectivas.

- Asegurar la coherencia de las pruebas de conocimientos con la estructura del Espacio Europeo de Educación Superior.

El documento también aborda un aspecto crucial en el sistema de las oposiciones: los tribunales. Señala la poca transparencia en la designación de sus miembros, ya que no depende de un proceso abierto o concurrencial. También aboga por "avanzar en la profesionalización y cualificación técnica de los órganos de selección".

Como antes he señalado esta reforma será lenta, incluso se plantea que los cambios serán más visibles a partir de la Oferta de Empleo Público de 2025. Además, hay que tener en cuenta que desde que aparece la OEP en el BOE hasta que se publican las diferentes convocatorias de las oposiciones pueden transcurrir tres años. Pero, a pesar de este amplio horizonte, son varias las acciones que se pueden acometer. 
La primera de ellas sería la adecuación de los temarios a las competencias que los bibliotecarios y archiveros desarrollan en sus puestos de trabajo. Es cierto que este esfuerzo se ha realizado y ya aparecen muchos temas de tecnologías y asuntos profesionales actuales. Pero aún con todo, es necesario un análisis más pormenorizado de los temas que figuran. Estos temarios deben reflejar conocimientos que sean necesarios en el quehacer de los profesionales. También habría que potenciar temas que puedan conllevar una visión personal de los candidatos. Un trabajo muy loable para reseñar es el que realizó el Consorcio de Bibliotecas Universitarias Andaluzas en el que identificaron cinco competencias clave a partir de las cuales idearon temarios y pruebas. También sería interesante la participación en el diseño de estos temarios de agentes externos tales como las universidades o las asociaciones profesionales.

La segunda acción sería que los tribunales, en aquellas oposiciones que los requieran, diseñen cuestionarios muy bien formulados, actualizados y que reflejen con rigor los temarios publicados. En ocasiones, algunos de estos cuestionarios parece que se han realizado de forma improvisada y contienen preguntas nada pertinentes o sobre aspectos que tienen poca importancia en la actualidad.

La tercera medida afecta directamente a la universidad. En las últimas oposiciones realizadas en los cuerpos de facultativos de bibliotecas y archivos se observa una baja participación en estos procesos y numerosas plazas que han quedado desiertas. Este último problema ha quedado reflejado en el documento del Ministerio al que estamos haciendo referencia. Entre los cuerpos y escalas con un porcentaje de cobertura de plazas inferior al $70 \%$, figura el Cuerpo de Facultativo de Archiveros, Bibliotecarios y Arqueólogos con tan solo un 50\%. Son pocos los titulados en Información y Documentación que se plantean opositar. Las causas pueden ser variadas: desconocimiento de estos procesos; una procedencia social de estos estudiantes media-baja que conlleva que ellos y sus familias no puedan soportar la dedicación exclusiva al estudio durante uno o dos años; y por último, puede afectar el hecho que en los actuales grados se ha disminuido mucho el estudio teórico, primando los contenidos prácticos, y esto ha provocado que la capacidad de estudio de los egresados no sea la más adecuada para afrontar con éxito oposiciones con un gran número de temas en sus convocatorias.

El futuro de las bibliotecas y de los archivos depende en gran medida de su personal. Su selección como hemos visto está en proceso de cambio y es necesario un debate en la profesión. Desde estas páginas contamos con las opiniones y puntos de vista de excelentes profesionales que conocen de cerca las convocatorias de oposiciones. Por orden alfabético, intervienen: María Trinidad Alonso Moya. Subdirectora de Biblioteca y Proyectos, Universidad de Jaén; Javier Argento. Opositor; Mateo Macía Gómez. Archivero Bibliotecario de las Cortes Generales y Director de Documentación, Biblioteca y Archivo del Congreso de los Diputados; Isabel Moyano Andrés. Subdirectora General del Libro de la Comunidad de Madrid; Clara Ortega Villanueva, Subdirectora General de Coordinación Bibliotecaria del Ministerio de Cultura y Deporte y Ricard Pérez Alcázar, Jefe de Área de Gestión Documental y Administración Electrónica de la Subdirección General de los Archivos Estatales del Ministerio de Cultura y Deporte.

\section{Cuestionario}

\section{1. ¿Cómo considera que será la evolución de las convocatorias de oposiciones para bibliotecas y archivos en los próximos años?}

María Trinidad Alonso Moya (Biblioteca universitaria) 
En mi opinión no creo que vayamos a ver grandes innovaciones en los próximos años. La Administración Pública es un engranaje muy complejo y requiere un esfuerzo colectivo unánime para modificar los procesos. Los cambios, cuando llegan, suelen deberse a la obligación de aplicar una ley que generalmente lleve aparejada financiación, y por tanto impulsada por una administración superior.

Por otra parte, no podemos olvidar, al menos en las bibliotecas universitarias que es mi ámbito, que formamos parte de organizaciones que tiene una gran diversidad de personas y que el proceso de selección se negocia con el equipo de gobierno y los representantes sindicales. La participación de los servicios de biblioteca se reduce en muchos casos a proponer un temario. La Universidad busca uniformar los procesos selectivos y eso deja muy poco margen a la especificidad de cada profesión o a las ideas innovadoras de un directivo o directiva de biblioteca. Creo que la evolución vendrá primero en los procesos selectivos de promoción interna, donde hay más cabida para aplicar los perfiles de cada puesto y las competencias, sean estas estratégicas de la organización o específicas del puesto.

Pero no toda la responsabilidad está en nuestras instituciones, hemos debatido largamente sobre competencias profesionales y oposiciones en estos años y hemos redactado documentos realmente buenos. Creo que ahora que sabemos el estado de la cuestión es el momento de hacer un esfuerzo de creatividad y un cambio del modelo de bibliotecario que deseamos conquistar para nuestras organizaciones.

\section{Javier Argento Vidal (Opositor)}

Ante la situación actual sólo puedo esperar que sea buena y constante. A nivel estatal es cierto que desde 2007 hasta 2015 tanto bibliotecas y archivos sufrimos una sequía muy grande de convocatorias, y que actualmente desde la Oferta de Empleo Público de 2015 ha habido plazas de acceso libre para bibliotecarios y archiveros en el Ministerio de Cultura (excepto la OEP de 2020).

Esta situación de oposiciones regulares y constantes es una muy buena noticia, porque nos sirve para prepararnos y poder organizarnos en los años que van a venir. Lo que espero que no ocurra, es lo que suele pasar en bibliotecas de las comunidades autónomas o universitarias, donde el panorama es distinto: ni convocan con una regularidad constante que a lo mejor puede ser de diez años en diez años, ni el número es excesivamente alto para el tiempo transcurrido entre convocatoria y convocatoria. Por poner un ejemplo práctico, el caso de la Escala de Ayudantes, Archivos, Bibliotecas y Museos de la Universidad Nacional de Educación a Distancia (UNED) en la Convocatoria de 1996, el número de plazas fue de 6; en la convocatoria de 2004, 9; y en la convocatoria de 2020, 4 plazas de estabilización.

\section{Mateo Macía Gómez (Biblioteca parlamentaria)}

Lo mejor sería que se evolucionara hacia sistemas menos basados en la memoria y en el número de temas (algo que todavía se considera importante en oposiciones para cuerpos superiores) y más en la capacidad y las habilidades. Hay y habrá algunas resistencias, pero los sistemas de selección irán cambiando de forma progresiva. 
Isabel Moyano Andrés (Coordinación bibliotecas de Comunidad Autónoma)

En este momento no parece que los procesos selectivos tal como los conocemos vayan a sufrir grandes transformaciones. Creo que probablemente la transformación debe incluir otros sectores y debería producirse un cambio en el conjunto de los sectores implicados: en primer lugar, tratar de ajustarse lo más posible a los nuevos tiempos, cambiar la formación en las escuelas de Biblioteconomía, poner en relación directa las oposiciones de las distintas administraciones con la práctica profesional de cada una de ellas y tener en cuenta la experiencia profesional cuando se hacen oposiciones enfocadas a la promoción interna. Podría ser interesante consensuar entre las distintas administraciones un modelo común de oposición que facilitara que los opositores puedan presentarse a convocatorias diferentes con una base común. Es decir, trabajar en impulsar convocatorias que presenten modelos iguales en aquellos aspectos comunes a las diferentes administraciones. Este modelo podría impulsarse y trabajarse a través del Consejo de Cooperación Bibliotecaria donde está representada toda la tipología de bibliotecas.

\section{Clara Ortega Villanueva (Coordinación bibliotecas del Estado)}

Considero que estamos en un momento esperanzador en cuanto a las oposiciones de los cuerpos y escalas de facultativos, ayudantes y auxiliares de bibliotecas y archivos de la Administración General del Estado (AGE), ya que en las Ofertas de Empleo Público que aún no se han resuelto, que son las correspondientes a los años 2018, 2019, 2020 y 2021 hay prevista la convocatoria de un total de 747 puestos.

Los puestos de las ofertas de 2018 y 2019 ya se han convocado (401 plazas) y los exámenes se están celebrando ya.

Aunque lo cierto es que en estas cifras se incluyen algunos puestos de auxiliares y ayudantes de museos que no están desagregados, se ha puesto de manifiesto que la AGE está apostando por estos cuerpos, por lo que creo que es un buen momento para los opositores de bibliotecas y archivos.

\section{Ricard Pérez Alcázar (Coordinación archivos del Estado)}

En cuanto a la periodicidad y el volumen, esperamos mantener el carácter anual y un número de plazas suficientemente atractivo como para que los opositores perciban que se trata de un proceso selectivo consolidado y una oportunidad merecedora, por lo tanto, de la inversión de tiempo y dedicación necesarios.

En cuanto a la posible evolución de los requisitos y trámites del proceso selectivo, es previsible que se tienda a reducir el protagonismo de lo memorístico.

Esta posible variación en el aspecto memorístico se relaciona con las tendencias observadas en las esferas académicas, donde la memorización se sustituye por una formación en competencias. En este sentido, la actual LOMLOE ya ha iniciado este camino, en el que el objetivo es generar destrezas y un mejor conocimiento del contexto. Por lo tanto, siguiendo este discurso y en consonancia con sus objetivos, sería un ejercicio de armonía y normalidad que los objetivos de los procesos selectivos también se orientaran a una medición de esas destrezas, por encima de la capacidad memorística de los opositores. 
Por último, y en correlación con la transformación digital de nuestra sociedad y servicios, también podría intuirse que esa necesaria destreza tecnológica empiece a considerarse un elemento primordial y en consecuencia se introduzca en estos procesos selectivos como un nuevo requisito.

2. ¿Qué opinión tiene sobre la pertinencia de los ejercicios actuales de las oposiciones de las tres escalas de bibliotecas y archivos (auxiliares, ayudantes y facultativos)? ¿Cómo podrían mejorarse?

\section{María Trinidad Alonso Moya (Biblioteca universitaria)}

Los bibliotecarios tenemos una profesión muy poco conocida, que requiere autoaprendizaje constante y predisposición al cambio. Las convocatorias que proponemos se basan en conocimientos teóricos y prácticos muy específicos. Buscamos gente muy preparada teóricamente y eso se refleja en las convocatorias, con temarios especializados que no tienen reflejo en el contexto laboral.

Necesitamos gente que sea capaz de recuperar información fiable, veraz, especifica y pertinente. Necesitamos que entiendan el trabajo que se realiza en una biblioteca, cómo se ordena el conocimiento y se pone a disposición de un investigador. Necesitamos candidatos abiertos al cambio y adaptados a las nuevas tecnologías, que comprendan la importancia de ser intermediarios del conocimiento. Esas son algunas de nuestras mejores habilidades y no las preguntamos en las convocatorias.

En resumen, creo que los contenidos que exigimos están bien, pero los preguntamos mal. El cambio en las convocatorias tiene que ir dirigido a encontrar a los mejores candidatos que tengan las habilidades y competencias que necesita un bibliotecario: conexión con el usuario, habilidad tecnológica, capacidad de autoaprendizaje, autonomía intelectual, y eso se puede preguntar de otra manera. Los comportamientos observables que describimos dentro de las competencias profesionales en nuestros perfiles, pueden ser el camino. Eso y un grupo de trabajo nacional que se ponga a pensar cómo.

\section{Javier Argento Vidal (Opositor)}

Creo que debemos partir de la idea de que todo se puede mejorar. A nivel estatal el Consejo de Cooperación Bibliotecaria (CCB) elaboró un documento con una interesante propuesta sobre los temarios de oposiciones a bibliotecas para el Ministerio de Cultura y Deporte, en el que se hablaba de cambiar diversas pruebas, ampliándolas o reduciéndolas. En él se podía ver la intención del personal funcionario de querer cambiar los procedimientos. El mismo trabajo que ha realizado el CCB para las oposiciones estatales, se debería realizar en las comunidades autónomas, locales y universitarias, o al menos tener en cuenta el trabajo del CCB para intentar aplicarlos a sus convocatorias.

No obstante, no debemos olvidar que los ejercicios de las oposiciones son propuestos y redactados por los tribunales. En este sentido, los tribunales deberían conocer mejor el trabajo 
de los niveles que están evaluando, es decir, que se adecuen a la convocatoria y al sentido común.

Algunas de las soluciones que deberían proponerse son:

- Que el tribunal esté formado en su mayoría por el mismo nivel de personal al que se evaluará. Ello nos va a llevar a tener un tribunal que tiene más contacto con las funciones que examina. Por ejemplo: para un examen de auxiliares de biblioteca, poco sentido tiene que examine un facultativo de bibliotecas, ya que no comparten funciones.

- Formación específica para los tribunales. Poder aprobar una plaza de bibliotecario o archivero, no nos faculta como personas para saber plantear un examen o corregirlo. Es cierto que sabremos del tema, pero no es necesario que sepamos evaluar correctamente. Para ello debería haber alguna formación desde el INAP o por pedagogos con cursos específicos en los que se plantearan capacidades específicas de evaluación y corrección para tribunales.

- Criterios claros y transparencia. El problema de las oposiciones es una falta de transparencia de los criterios que se utilizan a la hora de corregir un examen, y cuando se publican suelen ser vagos o muy generales (Ejemplo de la UNED: http://portal.uned.es/pls/ portal/docs/PAGE/UNED MAIN/LAUNIVERSIDAD/VICERRECTORADOS/GERENCIA/RECURSO S\%2OHUMANOS/SECCION\%2OPAS/OPOSICIONES_Y_CONCURSOS/PERSONAL_FUNCIONARI O/OPOSICIONES_LIBRES/ESCALA\%20DE\%20AYUDANTES\%20DE\%20ARCHIVOS\%20BIBLIOTEC A 20 \% $\% 20 M U S E O S / A C U E R D O \% 20 C R I T E R I O S \% 20 S E G U N D O \% 20 E J E R C I C I O \% 20 A Y U D A N T E S \% 2$ oBIBLIOTECA\%202021.PDF) Además, creo que una buena práctica de transparencia que ayudaría a los opositores a prepararse mejor las oposiciones sería que el tribunal publicara tanto los cuestionarios, como las soluciones. Por obligación se publican las plantillas, pocas veces los cuestionarios, y en la inmensa mayoría de casos no se publican los otros tipos de supuestos. Al respecto, algunas administraciones como los últimos tribunales del Ministerio de Cultura y Deporte y la Comunidad de Madrid están publicando los enunciados de las preguntas. También me gustaría reseñar, la iniciativa que se ha tenido en el Ministerio de Cultura en las oposiciones de Ayudantes y Facultativos de colgar los curriculum vitae de los tribunales, creo que es uno de los ejemplos de transparencia que necesitamos.

\section{Mateo Maciá Gómez (Biblioteca parlamentaria)}

El sistema de selección actual no se corresponde con el sistema educativo ni con los métodos de selección de personal de otros países o las organizaciones internacionales. Es casi una excepción. El régimen de los funcionarios españoles es también muy peculiar. La evolución lógica es hacia sistemas de selección que tengan en cuenta el acceso casi universal que hay actualmente a la información y los métodos educativos en la Universidad.

\section{Isabel Moyano Andrés (Coordinación bibliotecas de Comunidad Autónoma)}

Creo que las oposiciones deberían avanzar hacia modelos más prácticos y el desarrollo de procesos que permitan descubrir las habilidades y las capacidades de los opositores. Debería trabajarse en modelos flexibles de oposiciones que permitan la adecuación rápida de temarios y ejercicios a la rapidez con la que se producen los cambios en el mundo de la información. 
Considero que habría que avanzar hacia modelos menos teóricos. Tal vez sea en estos ejercicios prácticos donde deba hacerse un esfuerzo de adecuación a las necesidades de cada biblioteca y orientarlos a las necesidades reales de trabajo de las bibliotecas.

\section{Clara Ortega Villanueva (Coordinación bibliotecas del Estado)}

Tanto el número de ejercicios como su contenido vienen condicionados por la Subdirección General de Planificación de Recursos Humanos y Retribuciones del Ministerio de Política Territorial y Función Pública, que es a quien corresponde revisar e informar sobre las convocatorias.

En el último año, se han simplificado en la medida de lo posible algunos de los ejercicios, permitiendo cierta elección de contenidos por parte del opositor u opositora y reduciendo los ejercicios que versan sobre el mismo temario.

En mi opinión, se debe avanzar hacia ejercicios más personalizados y enfocados a demostrar que el/la candidata/a tiene las competencias necesarias para el desempeño de los puestos ofertados en cada caso.

También es necesario tener en cuenta las competencias que se exigen a los/as profesionales de las bibliotecas hoy en día y ser capaces de introducirlas en los ejercicios. No es fácil, pero hay que intentarlo.

No obstante, considero que los ejercicios actuales son adecuados, aunque susceptibles de mejora, puesto que los nuevos funcionarios que se incorporan tras haber superado las oposiciones tienen una formación excelente y unas capacidades que se ajustan a lo que se requiere de ellos en sus puestos de trabajo.

\section{Ricard Pérez Alcázar (Coordinación archivos del Estado)}

Al margen de lo ya expuesto, la exigencia que el opositor percibe al abordar la preparación del proceso selectivo y el volumen del temario son aspectos que contribuyen a que mayoritariamente se tienda a optar por el aparentemente camino más sencillo que brinda la entrada en la Administración a través del Cuerpo de auxiliares.

De este modo se producen dos efectos perniciosos: en primer lugar, las plazas de auxiliares se cubren por personal sobrecualificado que, con todo el derecho (por supuesto), transitoriamente, solo ocuparán esos puestos de trabajo el tiempo que tarden en buscar las plazas de promoción interna. Consecuente y reiteradamente, a medio plazo irán quedando vacantes las plazas que se vayan abandonando; en segundo lugar, el corto número inicial de opositores que se presentan a los Cuerpos de ayudantes y facultativos son insuficientes para asegurar que se cubran todas las plazas ofertadas.

Evidentemente, un ejercicio tipo test es visto como un objetivo más al alcance que el desarrollo por escrito de un volumen de contenidos que no pueden ser respondidos más que a través de un estudio planificado.

En cuanto a los ejercicios de ayudantes y facultativos, la gestión y resolución de los supuestos prácticos es uno de los principales escollos. Por ello, desde esta Subdirección General se está aportando ya mayor información sobre esta parte del ejercicio selectivo. A través de nuestra web, en el apartado de la última OEP cerrada (de 2017) ya se ha incorporado información sobre 
los supuestos reales que se asociaron al caso práctico y al idioma. Esta información debería servir para entender mejor la dimensión de estos ejercicios.

3. ¿Cuáles son sus valoraciones sobre la adecuación de los temarios actuales de los ejercicios de las oposiciones de las tres escalas de bibliotecas y archivos (auxiliares, ayudantes y facultativos) a los puestos ofertados? ¿Cómo podrían actualizarse?

\section{María Trinidad Alonso Moya (Biblioteca universitaria)}

He visto temarios para auxiliares de biblioteca donde se pregunta por protocolos de Internet que solo van a usar en su vida profesional a nivel de usuario, no tiene sentido. Quizá habría que preguntar por lo que se hace en una biblioteca y preguntar con sencillez.

Los temarios se tienen que simplificar y eso es un trabajo muy difícil. No es que hagamos temarios más sencillos, que también, es que hagamos temarios más lógicos y más conectados con el trabajo real.

Las organizaciones, a su vez, deben hacerse cargo de formar a los nuevos bibliotecarios en los aspectos concretos de su sistema o institución, tanto como se ocupan de formar a los bibliotecarios veteranos en una profesión que requiere aprendizaje durante toda la vida.

\section{Javier Argento Vidal (Opositor)}

El mismo documento al que he hecho referencia en el apartado anterior se contempla una actualización de los temarios de oposiciones. En muchas de nuestras oposiciones nos encontramos con temas desfasados, algunos que se repiten, otros vagos a la hora de expresarse, etc. Esto supone un esfuerzo a la hora de estudiar o redactar, ya que, si el enunciado no es claro, nuestras respuestas tampoco lo serán.

Aquí me gustaría hablar de la disparidad de temas a los que nos enfrentamos si un opositor decide presentarse a varias convocatorias a la vez. Creo que una buena solución que debería nacer desde un organismo superior como FESABID o el Consejo de Cooperación Bibliotecaria sería la redacción de unos temas comunes a todas las bibliotecas, y dentro de cada tipo de biblioteca establecer unos mínimos y unos máximos de temas. Por ejemplo, para una biblioteca local: 10\% legislación, 50\% biblioteconomía, 20\% fomento lector y servicios culturales, 10\% información local. Para ello se debería redactar enunciados de temas en los que los responsables de formación de tribunales y los mismos pudieran acceder a ellos, así como los opositores. Otro ejemplo, en la Comunidad de Madrid hay actualmente hay ocho universidades públicas, las cuales ofertan plazas de ayudantes y auxiliares de bibliotecas. Pues debería haber un mínimo de temas exactamente iguales entre las ocho universidades, aparte, claro de los temas propios como estatutos o cartas de servicios de la biblioteca propia.

Si propiciamos esta iniciativa, los opositores podemos presentarnos a más convocatorias, con temas mejor estructurados y preparados, y no vernos en la necesidad de ir cambiando enunciados, párrafos, etc. para cada convocatoria específicamente. 
Mateo Maciá Gómez (Biblioteca parlamentaria)

La cuestión para mi es si es necesario que haya tres escalas de bibliotecas y archivos. En mi opinión esto constituye un verdadero anacronismo, no se corresponde con las necesidades profesionales en la sociedad de la información. En cualquier caso, si se van a mantener, lo primero que habría que definir es cuales son las funciones de estas tres escalas hoy y a partir de eso los mecanismos de selección. En la actualidad los tres temarios tienen el mismo contenido. Se diferencian por la profundidad y el número de temas.

Yo preferiría evolucionar hacia un sistema de meritoriaje en el que hubiera una selección de entrada y una carrera administrativa basada en el trabajo y las capacidades desarrolladas.

En cualquier caso, los cuerpos "facultativos" deberían acreditar en la actualidad capacidad de gestión económica y de personal y de comunicación pública.

Isabel Moyano Andrés (Coordinación bibliotecas de Comunidad Autónoma)

Creo que los temarios deberían reducirse y eliminar partes tradicionales de los temarios que han quedado completamente obsoletas y que tienen poco que ver con el trabajo real de las bibliotecas.

Las oposiciones de ayudantes y facultativos adolecen de ejercicios que incidan más en cuestiones de gestión y que por en este momento están muy diluidas en los temario y ejercicios prácticos. Por otro lado, hay administraciones donde las convocatorias de oposiciones no están separadas en función del área de especialidad, es decir la oposición es conjunta para archivos, bibliotecas y museos. En mi opinión esto es un error dada la especialización existente en cada una de las especialidades.

\section{Clara Ortega Villanueva (Coordinación bibliotecas del Estado)}

En el último año, en las convocatorias de la AGE se ha realizado un importante esfuerzo para adecuar los temas a las nuevas necesidades de los profesionales.

Se han tenido en cuenta para ello algunos estudios y trabajos anteriores, como los realizados en el seno de la Biblioteca Nacional de España y las propuestas sobre temarios realizadas por el Grupo de trabajo sobre perfiles profesionales del Consejo de Cooperación Bibliotecaria.

Si bien el número de temas también está condicionado por lo determinado por la Subdirección General de Planificación de Recursos Humanos y Retribuciones del Ministerio de Política Territorial y Función Pública, se han modificado los temarios de ayudantes y facultativos de bibliotecas, se ha introducido la opción de elección de temas en algunos ejercicios y escalas y además en algunos cuerpos como el de ayudantes de bibliotecas se ha reducido el número de temas.

\section{Ricard Pérez Alcázar (Coordinación archivos del Estado)}

Ante esta cuestión, como primer inconveniente se parte de la premisa de que no existe una norma que circunscriba y detalle las funciones de los diferentes cuerpos técnicos de archiveros, a pesar de que existe una profusa producción bibliográfica sobre la temática de la adecuación de 
los puestos de trabajo. Partiendo de ahí, todo intento de asociar los temas a las funciones podrá ser cuestionado, cuestionable y parecer inadecuado. En este sentido la formalización de un documento con los contenidos laborales (actividades y tareas) asociados a cada uno de los Cuerpos facilitaría la transparencia en la adecuación de los contenidos exigidos a cada cuerpo. Pero como no se dispone de esa herramienta, la primera piedra de toque queda fuera del propio proceso.

En el ámbito de los archivos es habitual escuchar opiniones sobre el excesivo número de temas relacionados con materias históricas y el reducido número de los relacionados con las nuevas tecnologías. Y la respuesta habitual ante estos comentarios es recordar que, en el caso de los Archivos Estatales, siete de los ocho Archivos son de carácter histórico. También es perceptible, por otro lado, que en las últimas Ofertas de Empleo Público (OEP) cada vez es mayor el porcentaje de nuevos funcionarios que tienen como primer destino centros de archivo fuera del Ministerio de Cultura y Deporte. Quizás este último apunte invitaría a medir la conveniencia de añadir un porcentaje de temas relacionados con este tipo de centros y sus funciones específicas. Desde esta Subdirección General de los Archivos Estatales, se percibe la necesidad de consensuar un temario que sea producto del acuerdo técnico con otras Administraciones y las asociaciones profesionales. Este temario normalizaría los contenidos y el material de estudio necesarios, además de simplificar el esfuerzo requerido a los opositores cuando se deciden presentarse ante diferentes Administraciones. La posibilidad de que el estudio de un temario sirviera para diversas tentativas ante diversas Administraciones probablemente significaría un aumento numérico en los listados de admitidos.

4. ¿Qué formación cree que es la más adecuada para las oposiciones de las tres escalas de bibliotecas y archivos (auxiliares, ayudantes y facultativos)? Indique si tiene alguna opinión sobre la formación de las asociaciones profesionales, universidades y academias.

\section{María Trinidad Alonso Moya (Biblioteca universitaria)}

Sin lugar a duda yo creo que el grado de Documentación o de Biblioteconomía y Documentación es el camino más llano para llegar a ser bibliotecario, documentalista o archivero. La mayoría de los temas ya se ven en la carrera, tienes el contexto y solo hay que ponerse a estudiar.

Creo que para la parte práctica las academias y asociaciones profesionales funcionan bien. Pero en mi opinión, el candidato tiene que preparar los temas leyendo mucho. Al menos en las bibliotecas universitarias nunca han triunfado los temarios publicados.

Y en último lugar, creo que las Facultades de Documentación tienen un reto respecto a la formación de sus estudiantes para la práctica laboral. Si el currículum es tan extenso y diverso que no es posible, siempre se puede crear un experto o un máster de práctica bibliotecaria, no digo que tengan que ser habilitante para ejercer, pero que pueda ser referente.

\section{Javier Argento Vidal (Opositor)}

No creo que ninguna formación o carrera universitaria nos prepare específicamente para el mundo de las oposiciones. En nuestro ámbito muchos de los opositores reciben la formación a 
través de academias, cursos de asociaciones o preparadores, que les ayudan a iniciarse en los temas, la catalogación, la ordenación o los supuestos.

A pesar de ello, creo que no debemos olvidar el actual Grado de Información y Documentación que se imparte en varias universidades españolas. En teoría, este Grado es el que más nos acerca al mundo de las oposiciones a bibliotecas y archivos, y así al menos ha sido en mi caso, a la hora de saber dónde buscar información, redactar los temas de oposiciones, preparar ejercicios como catalogación o supuestos, o simplemente saber de qué tratan los temas.

No obstante, no estaría de acuerdo con el afirmar que nuestro Grado deba preparar exclusivamente para las oposiciones, pues entonces perderíamos la idea de que nuestro perfil profesional no puede encontrarse en empresas privadas. Lo que sí que creo que se debería potenciar en las Facultades de Documentación es conocer en qué consisten las oposiciones, conocer a personas que han aprobado, que están en proceso, ya fuera mediante charlas, talleres, etc.

Lo ideal es que en los últimos años del Grado se fueran formando grupos de estudiantes que quisieran opositar a bibliotecas y archivos, y ya se fueran preparando, tanto mentalmente, como a la hora de ir elaborando temarios o recursos para las oposiciones.

Como conclusión a esta pregunta, me gustaría plantear otra pregunta: ¿Podemos los opositores aprobar una oposición de bibliotecas o archivos sin recibir formación específica por parte de una academia o un preparador? Yo creo que no. Y aquí debería evaluar cómo están presentadas las oposiciones a Ayudantes y Facultativos, ya que no toda la gente tiene los mismos recursos.

\section{Mateo Maciá Gómez (Biblioteca parlamentaria)}

Para preparar las oposiciones tal como están ahora lo más cómodo es una academia. Lo más efectivo suele ser el trabajo individual con la ayuda de un preparador específico. La universidad no prepara para las oposiciones, proporciona unos conocimientos básicos que hay que adaptar al formato de oposiciones tradicionales.

Las asociaciones profesionales pueden tener un papel en la preparación, pero sus objetivos son otros.

\section{Isabel Moyano Andrés (Coordinación bibliotecas de Comunidad Autónoma)}

Creo que habría que diversificar también por el tipo de biblioteca al que se vaya a acceder. No es lo mismo una pequeña biblioteca municipal que una biblioteca universitaria o de investigación. Creo también que las facultades de biblioteconomía en ocasiones olvidan materias más generalistas tipo literatura o historia que son imprescindibles para trabajar en bibliotecas con patrimonio bibliográfico importante, bibliotecas que son abundantes en nuestro país o para poder hacer trabajos de prescripción y fomento de la lectura en las bibliotecas públicas

También considero desde la perspectiva profesional de la que me ocupo en este momento que las facultades de biblioteconomía han hecho una clara opción por los temas que tienen más que ver con los lenguajes documentales y la búsqueda en internet, mientras que no existen materias que incidan en prácticas profesionales menos teóricas y que forman parte del trabajo diario de una biblioteca pública, ofertando instrumentos que tengan que ver con el papel de los bibliotecarios como prescriptores de lectura, animadores a la lectura o a programar actividades para los diferentes sectores sociales. 
Creo que se incide mucho en cuestión de perfiles muy técnicos y muy especializado como el catalogador o el comunity manager.

No encuentro tampoco en los planes de estudio ninguna referencia a planes de fomento de la lectura o al conocimiento del sector del libro, sector que ocupa un lugar importante en el PIB.

\section{Clara Ortega Villanueva (Coordinación bibliotecas del Estado)}

En el caso de bibliotecas, la formación más adecuada es la que ofrecen las facultades de biblioteconomía, información y documentación. La mayoría de los contenidos de los temarios de las oposiciones se estudian en estas facultades y los supuestos prácticos de las oposiciones también suelen estar contenidos de alguna manera en los planes de estudios. En mi opinión, las facultades podrían motivar más a los estudiantes a la preparación de oposiciones, explicando los contenidos de los ejercicios y los puestos de trabajo que se pueden desarrollar en las administraciones.

Los cursos especializados de asociaciones profesionales y academias son también muy valiosos, sobre todo los que van enfocados directamente a la preparación de oposiciones puesto que, además de contribuir a la mejora de la formación, reducen la inseguridad de los opositores y los ponen en contacto con otras personas que están estudiando lo mismo que ellos. La colaboración entre los opositores puede ser muy ventajosa para todos si cooperan en compartir recursos e información, preparar temas en común, etc.

Los opositores que han estudiado otras disciplinas o provienen de otros campos y después se forman en materia bibliotecaria, pueden aportar una visión diferente, más enriquecedora, que valoran los tribunales y también se valora en el entorno laboral.

\section{Ricard Pérez Alcázar (Coordinación archivos del Estado)}

Las asociaciones profesionales, universidades y academias ofrecen una formación inicial que asegura un punto de partida. El contenido de los temas puede recabarse efectivamente a través de estos agentes. Al fin y al cabo, los tiempos variables de escritura de que se dispone para desarrollar cada uno de los temas en cada ejercicio requieren que de manera planificada se dedique un tiempo intransferible para personalizar esos mismos contenidos. La rapidez en la escritura, la capacidad memorística para retener la estructuración de capítulos o la capacidad de síntesis en el discurso son elementos que varían y que, en consecuencia, pueden significar que un mismo texto no sea válido para diferentes opositores.

De ahí que en la sección de archivos es habitual la figura del preparador, que es quien habitualmente ayuda a corregir desviaciones en los esfuerzos, orientar en los repartos de tiempo y ayudar a personalizar los citados contenidos.

No existe una respuesta definitiva sobre cuál es la vía más adecuada. En lo que sí cabría insistir es que justamente ese tiempo invertido en la personalización de los temas es el que generará un poso básico de confianza y seguridad que acompañará positivamente al opositor.

5. Cada vez con más frecuencia son menos los opositores que se presentan a los cuerpos, sobre todo superiores, de bibliotecas y archivos. Por ejemplo, en la última convocatoria del Ministerio 
de Cultura y Deporte que se ofertan 22 plazas libres de facultativos de bibliotecas y 46 plazas de archivos tan solo 181 personas han firmado la oposición para bibliotecas y 288 para archivos ¿A qué cree que es debido? ¿Cómo considera que se podría motivar a los posibles candidatos a presentarse?

\section{María Trinidad Alonso Moya (Biblioteca universitaria)}

A menudo me responden a esa preguntan con algunos razonamientos muy lógicos, por ejemplo, las oposiciones no tienen continuidad en la oferta, no salen todos los años, así que muchos candidatos no saben cuánto tiempo tendrán que invertir.

Por otro lado, no hay una única convocatoria con un tipo de examen y un temario concreto, sino multitud. Es decir, tendrás que decidir si quieres ser bibliotecario del ministerio, de tu comunidad autónoma, de tu municipio, de un centro de investigación o de una universidad y, ojo, según de cual, porque cada una de las administraciones hacemos nuestros propios perfiles y nuestras propias convocatorias, de forma que alguien que decide ser bibliotecario tiene muchas más incertidumbres que certezas.

Creo que las administraciones tenemos mucha responsabilidad en desincentivar a los participantes, la solución: continuidad en la oferta de plazas, temarios comunes, pruebas similares y movilidad entre administraciones.

Y por último insistir en lo anterior, modificar el sistema de selección. Simplificar las convocatorias, aunque eso conlleve un esfuerzo de formación de los nuevos bibliotecarios que aprueben por parte de la administración convocante.

\section{Javier Argento Vidal (Opositor)}

Por un lado, creo que la profesión aún no es del todo bien conocida, y no me refiero a nivel general, sino entre los propios estudiantes de Grado y Máster. Es cierto que durante nuestra formación conocemos diferentes bibliotecas y archivos de diferentes administraciones públicas en las que nos enseñan las colecciones y servicios, pero en contadas ocasiones los propios trabajadores nos proporcionan información de cómo llegaron a esos sitios: qué oposición fue, cómo se la prepararon, cómo fue el proceso, etc. Si desde nuestras facultades se animara más a presentarnos, estoy seguro de que se animaría más gente a presentarse a las oposiciones de ayudantes y facultativos.

Además, creo que debemos analizar el panorama actual de las oposiciones. Por un lado, salen pocas convocatorias, por otro lado, salen en diferentes administraciones. El hecho de no poder tener temarios comunes o que coincidan pocos, hace que no nos podamos presentar a tantas oposiciones como quisiéramos o pudiéramos. También hay que tener en cuenta que este año 2021 ha habido un cambio considerable en los temarios, tanto de ayudante como de facultativo, por lo que habrá gente que le ha sorprendido y no habrá podido prepararse los temas adecuadamente. Tampoco en sí el procedimiento ni el temario es el más atractivo del mundo. Es inviable, y poco práctico, pedir tantos temas en estas oposiciones (Facultativos de Bibliotecas del Ministerio de Cultura: 127 temas, o la de Archiveros-Bibliotecarios de Cortes Generales con 168 temas), en comparación a la recompensa final.

Además, en otras oposiciones como las universitarias nos encontramos con oposiciones del tipo concurso-oposición, por lo que las personas que no gozamos de experiencia profesional (llevo más de cuatro años de becas para postgraduados en formación de biblioteconomía que no me 
cuenta para nada), no podemos hacer nada salvo intentar sacar una nota alta en la fase de oposición. Por poner otro ejemplo, para la Escala de Ayudantes de Archivos, Bibliotecas y Museos de la Universidad de Valencia, la fase de concurso es prácticamente un $40 \%$ del sistema selectivo. Creo que también habría que tener en cuenta la poca retribución que cobra un funcionario del cuerpo de bibliotecas y archivos del Ministerio de Cultura y Deporte, comparado con otras oposiciones como el Ayuntamiento de Madrid o las universidades, donde la misma escala y nivel, son mayores que las del Ministerio. Por último, no creo que motive mucho ver como sistemáticamente se van quedando desiertas.

\section{Mateo Maciá Gómez (Biblioteca parlamentaria)}

La desbandada obedece a varios factores. Por citar algunos:

- Dispersión de la formación. Hoy hay una Facultad de Ciencias de la Documentación en la Complutense, y unos cuantos grados en información y documentación en otras universidades que se imparten en varias facultades y con planes de estudios muy diferentes entre sí. Los estudiantes al final no entienden muy bien que es lo que han estudiado y para qué sirve.

- Falta de perfiles profesionales claros. A muchas oposiciones puede presentarse cualquier graduado universitario, transmite la sensación de que para esto vale cualquiera.

- Decadencia de la cultura del papel impreso, que todavía es en gran parte la cultura de las bibliotecas.

- Pérdida de la relevancia social. Antes las de bibliotecario o archivero podían verse como profesiones "raras", pero de alguna manera necesarias por la vertiente de conservación del patrimonio histórico y cultural y la indudable función compensatoria de las bibliotecas públicas. Hoy ya no. Además, hay una escasísima presencia en las redes o los medios de comunicación.

Para motivar a los candidatos hay que establecer conocimientos y funciones profesionales definidas no solo en la Administración, sino también en la sociedad.

\section{Isabel Moyano Andrés (Coordinación bibliotecas de Comunidad Autónoma)}

Desgraciadamente no creo que esta situación se produzca exclusivamente en las oposiciones de bibliotecas. Creo que es una situación generalizada que afecta a muchos cuerpos de la administración. Considero que se trata de una tendencia entre los sectores más jóvenes de la población que no contemplan entre sus posibilidades laborales el trabajo en la administración accediendo a través de una oposición. Sus expectativas profesionales son muchas veces más apremiantes y no existe la valoración de la estabilidad laboral que tenía en épocas anteriores. Considero que se debería promocionar el empleo público como servicio público y fomentar el reconocimiento social del que goza en otros países de nuestro entorno.

En los niveles más altos de la administración deberían producirse mejores remuneraciones más acorde con la formación de los profesionales

Las facultades y escuelas de biblioteconomía tal vez podrían realizar jornadas formativas sobre salidas profesionales y que los bibliotecarios de distintas instituciones hablases de los trabajos que realizan. Podría ser interesante una colaboración con las distintas administraciones para que 
personal de las mismas diera charlas en las facultades y pudiera despejar dudas de futuros opositores, proporcionando una visión real de la práctica profesional.

Clara Ortega Villanueva (Coordinación bibliotecas del Estado)

Es cierto y estamos muy preocupados por ello, ya que en la Administración General del Estado necesitamos bibliotecarios y archiveros para el correcto desarrollo de nuestras funciones y necesitamos que estén bien preparados.

Las razones para esta falta de opositores son diversas:

En primer lugar, se trata de oposiciones con temarios muy largos, y con varios ejercicios, que pueden desanimar en un primer momento.

Además, existe una oferta escasa de preparadores que acompañen a los estudiantes y de cursos de preparación en asociaciones profesionales y academias.

Y por último otro motivo puede ser que, en el caso de la AGE, la convocatoria de oposiciones de archivos y bibliotecas tradicionalmente ha sido poco regular, combinando años con un número significativo de plazas convocadas con años sin convocatoria de plazas o con convocatorias muy limitadas. Esto hace que no haya un número estable significativo de estudiantes de oposiciones y que a las academias generalistas de preparación de oposiciones cueste apostar por la preparación para estos cuerpos y escalas.

Para motivar a los posibles candidatos, me gustaría decir que, aunque a primera vista parecen muchos contenidos los que hay que estudiar y aprender, normalmente las diferentes oposiciones y los ejercicios dentro de las mismas se basan en temarios muy similares, en los que gran parte del temario aprendido puede servir para las oposiciones de diferentes escalas, ofertadas por diferentes administraciones. Es importante hacer al principio un importante esfuerzo de comprensión e interiorización de los temarios para después poder aprovechar los conocimientos ya adquirirlos y dirigirlos a las diferentes oportunidades que se presentan.

Además, en estos momentos hay poca competencia, en comparación con hace algunos años, por lo que el esfuerzo invertido en la preparación de oposiciones tiene una correlación mayor con la posibilidad de aprobar y obtener la plaza sin necesidad de competir.

\section{Ricard Pérez Alcázar (Coordinación archivos del Estado)}

En el caso de los archivos, no puede hablarse de que el número de opositores haya ido en descenso. Según nuestros datos, en el año 2014 los admitidos fueron 134; en 2016, 266; en 2017, 309; y en el último proceso selectivo (que incluye la OEP de los años 2018 y 2019), 315. En estos datos incluimos todos los admitidos: plazas libres y promoción interna.

La tendencia, por lo tanto, es un creciente aumento del número de personas que se sienten atraídos por este proceso. Ciertamente, lo que no existe es una correlación entre las plazas ofertadas y los opositores que se postulan a cubrirlas.

Una primera lectura de estos datos sería interpretar que la bolsa de personas interesadas es estable. Esta estabilidad abre, a su vez, una serie de interpretaciones que pueden ser contradictorias. Cabe la percepción de un nicho estable de interesados y de manera paralela la existencia de otro nicho de previsibles interesados que no se sienten concernidos ni atraídos.

En otras palabras, podríamos decir que hay ámbitos territoriales desde los que apenas llegan opositores y que este ámbito está estrechamente relacionado con que desde ciertos ámbitos académicos apenas nos llegan especialistas. Justamente, esta medición está siendo revisada en 
la actualidad a través de indicadores estratégicos que nos permitan detectar objetivamente desde qué universidades no se presentan opositores. Acotado el problema, la siguiente acción sería abordar cuál es nuestra relación con ellas y, si fuera el caso, variar o reelaborar nuestro discurso para trasladar correctamente la oportunidad profesional que puede representar entrar a trabajar en la Administración General del Estado (AGE).

Desde la AGE somos conscientes que la comparativa en cuanto a remuneración puede ser otro factor (creemos que menor) que invite a posibles opositores a optar por otras administraciones, pero ante este desequilibrio la oferta desde la AGE debe tender a explicitar la excelencia y el atractivo de los proyectos que se pueden ejecutar.

De todos modos, una conclusión en este aspecto sería que debemos insistir en la comunicación de las funciones y tareas que se asocian a estas plazas, para que los procesos selectivos se instalen como una salida profesional que desde las universidades se traslade a sus estudiantes.

6. Lamentablemente en las últimas convocatorias, sobre todo de los cuerpos superiores de bibliotecas y archivos, son bastantes plazas las que se quedan desiertas ¿considera que se podrían hacer alguna medida para paliar este problema?

\section{María Trinidad Alonso Moya (Biblioteca universitaria)}

Creo que respondo con la pregunta anterior y también creo que hay que estudiar. Parece una obviedad, pero no lo es, quien se quiera preparar unas oposiciones de bibliotecario tiene que estudiar mucho y tendrá que hacerlo toda la vida, porque es una profesión exigente y muy cambiante.

\section{Javier Argento Vidal (Opositor)}

Puede que este debate sea de los primeros foros donde ponemos este tema sobre la mesa, pero no creo que esté en nuestras manos solucionarlo. Debemos analizar este problema todos los agentes implicados: tribunales, opositores, funcionarios que acaban de aprobar, academias, universidad, preparadores, etc. No podemos culpar sólo a una parte la ecuación. Y más siendo los opositores, la parte más débil y perjudicada.

A nivel personal creo que los tribunales deberían evaluarse los procedimientos que están aplicando a los ejercicios. Publicándolos desde un primer momento, facilitaría a los opositores a entender mejor la oposición. A eso mismo, debemos exigir a los tribunales unos ejercicios que no excedan los niveles y los temarios de los grupos a los que están evaluando.

Sinceramente no creo que los opositores vayamos mal preparados a los exámenes, sobre todo cuando hablamos de los últimos ejercicios. Reducirlo todo a ello es muy simple. La situación de muchos de nosotros no es de dedicación absoluta a estudiar, ya que la inmensa mayoría tenemos un trabajo o beca en la que trabajar, cargas económicas que solventar, cargas familiares, hay personas que no pueden permitirse el lujo de una formación para la preparación, etc., sumado a todo ello los niveles de ansiedad y estrés que la propia oposición genera. 
Mateo Maciá Gómez (Biblioteca parlamentaria)

Habría que definir mejor los perfiles profesionales. En mi opinión, son básicamente dos: especialista en información y especialista en gestión de la información.

Hay que coordinar los planes de estudios de las diferentes titulaciones de grado para que sean homogéneos. Lo que los estudiantes deberían salir sabiendo es cómo circula y funciona la información en la sociedad y en distintos ámbitos concretos. Ello implica hoy necesariamente conocimientos tecnológicos, pero también sociológicos y de comunicación.

Saber buscar y valorar y, sobre todo, generar recursos de información adaptados a las formas de comunicación de nuestra sociedad, muy distintas de las de nuestros padres o nuestros abuelos.

Isabel Moyano Andrés (Coordinación bibliotecas de Comunidad Autónoma)

En primer lugar, debería haber continuidad en las convocatorias. Estas deberían convocarse todos los años o como mucho cada dos. Es cierto que esa continuidad no se ha tenido en los últimos tiempos y además muchas veces cada nueva convocatoria supone un cambio en los temarios. También como apunta antes en la falta de atractivo que tiene para los jóvenes el trabajo en el sector público.

Pero esto no puede restar importancia a otro de los motivos por los que se están quedando desiertas muchas de las plazas convocadas y es el bajo nivel de conocimientos de los opositores, a veces incluso deficiencias en la comprensión de lo que son los ejercicios y en las dificultades de redacción que se observan. No existe un trabajo de preparación de los temarios e incluso se utilizan temarios completamente obsoletos.

Existe también una falta de cultura general, carencias notables en redacción, gramática y ortografía, seguramente relacionada con la falta de conductas lectoras y que hace que se imponga el modelo de redacción que se observa en redes sociales, frases cortas y mensajes telegráficos. Sin embargo, esta situación no nos puede llevar a olvidar que los opositores de estos cuerpos están destinados muchas veces a desempeñar cargos directivos en las diferentes estructuras administrativas.

Pasar por alto estas carencias es condenar a la función pública a un descenso en la calidad del servicio. Probablemente en este sentido habría que incidir más en una educación secundaria y universitaria que formara en estos aspectos.

\section{Clara Ortega Villanueva (Coordinación bibliotecas del Estado)}

Algunas de las medidas no dependen de las administraciones y ya se han apuntado anteriormente: formación más orientada en las facultades y motivación de estudiantes, apuesta de las academias y asociaciones profesionales por la preparación de oposiciones y mayor motivación de los candidatos.

El Ministerio de Cultura y Deporte ya está llevando a cabo importantes medidas, como la adecuación de los temarios a las competencias, la introducción de opción de elección de temas o partes de ejercicios por parte del opositor, la reducción de temas en las oposiciones de Ayudantes de Bibliotecas, la supresión de un supuesto práctico en las oposiciones de Facultativo de Bibliotecas, el apoyo proporcionado por la BNE ofreciendo recursos de apoyo para opositores o el apoyo económico a asociaciones profesionales. 
Además, en las últimas convocatorias los tribunales pueden elaborar una lista de candidatos que, sin haber superado la oposición, podrían formar parte de una bolsa de trabajo.

\section{Ricard Pérez Alcázar (Coordinación archivos del Estado)}

Volviendo a la cuestión de las interpretaciones de la problemática sobre la cobertura de las plazas ofertadas, es un lugar común hablar de la falta de preparación de los opositores. No obstante, desde esta Subdirección General de los Archivos Estatales, a pesar de observarse que efectivamente se percibe que los contenidos transmitidos en las diferentes pruebas no son lo satisfactorios que se desearía, no se puede hablar como factor inicial de la falta de preparación. La premisa inicial se percibe desde el estadio anterior: el número de personas que pueden dedicar un tiempo de calidad a la preparación de una oposición ha descendido. Partiendo de esta situación, y al hilo de la respuesta anterior, podría pensarse que quienes disponen de ese tiempo tenderán a invertirlo en oposiciones que redunden en unas nóminas cuantitativamente superiores.

Los indicadores sobre el acceso al Cuerpos de facultativos nos informan que en los últimos procesos selectivos tan solo el $15 \%$ de los admitidos aprueban el primer ejercicio. Debemos plantear el problema desde lo que se puede aportar internamente para paliar esta situación.

Una posible línea de trabajo sería proporcionar una lista tasada de supuestos prácticos, en la línea de lo que ya se puede descargar respecto de la OEP 2017, para que el opositor pueda preparar con mayor criterio y seguridad este apartado.

Esta información no debería servir únicamente a los propios opositores sino también a aquellas unidades privadas (asociaciones, academias o preparadores particulares) que tradicionalmente se han encargado de participar en la conformación del material de estudio y trabajo.

De manera complementaria, desde hace algunos años ya se está proporcionando material bibliográfico asociado a cada uno de los capítulos del temario desde el Centro de Información Documental de Archivos (CIDA) en coordinación con las distintas bibliotecas de los Archivos Estatales (Bibliografía para oposiciones a Archivos).

Finalmente, aunque sea una reiteración, es necesaria mejorar la comunicación con los centros formadores (universidades) para trasladar convenientemente la oportunidad profesional que significa trabajar en los Archivos de la AGE.

\section{Conclusiones}

Las respuestas de los entrevistados ponen de manifiesto algunas de las cuestiones ya planteadas en la introducción. En primer lugar, una valoración positiva sobre el número de plazas que se están convocando para bibliotecas y archivos y una percepción general sobre la necesidad de que los sistemas de selección actuales evolucionen hacia modelos en los que se primen más las competencias y destrezas que los conocimientos memorísticos. Aunque, tal como se señala en una respuesta, a este respecto el margen de acción de los servicios de bibliotecas es más bien pequeño, ya que el diseño de las pruebas corresponde a otras instancias.

En segundo lugar, aunque se ponen de manifiesto ciertas mejoras en los temarios de las últimas oposiciones, se aboga por seguir actualizándolos para que tengan realmente 
correspondencia con el entorno laboral. A este respecto es imprescindible identificar muy bien los perfiles que se corresponden a cada cuerpo y escala y sus funciones. Se sugiere que los diferentes tipos de bibliotecas trabajen de forma coordinada a este respecto en el marco del Consejo de Cooperación Bibliotecaria. También algunas respuestas abogan por una mayor profesionalidad de los tribunales y una mayor transparencia en los procesos. Se pone de manifiesto como algunas instancias ya publican los currícula de los miembros del tribunal y los ejercicios planteados.

La formación de los opositores es otra cuestión que se ha debatido. Se considera que el Grado en Información y Documentación es la titulación universitaria más acorde, pero eso tampoco debe suponer que esta titulación solo se enfoque hacia el sector público. Lo que sí que parece claro es que desde la universidad se debe promocionar el empleo público y dar cuenta de sus posibilidades. Preparadores, academias y asociaciones profesionales contribuyen a esta formación. También algunas respuestas señalan la importancia en los niveles superiores de que el opositor sea quien se prepare los temas dando una visión personal de ellos.

Por último, también se ha puesto en evidencia el problema de que en los niveles más altos se estén quedando plazas desiertas y los pocos opositores que se presentan, fenómeno que sobre todo se dan en las oposiciones para bibliotecas, más que para las de archivos.

Para finalizar hay que constatar la importancia de esta temática. El personal de las bibliotecas y archivos públicos se va a renovar en los próximos años y es fundamental un debate en profundidad sobre su sistema de selección. El futuro de las bibliotecas y de los archivos depende principalmente de sus profesionales.

\section{Entrevistados}

María Trinidad Alonso Moya

Subdirectora de Biblioteca y Proyectos, Universidad de Jaén

Javier Argento Vidal

Opositor

Mateo Maciá Gómez

Archivero Bibliotecario de las Cortes Generales y Director de Documentación, Biblioteca y Archivo del Congreso de los Diputados

Isabel Moyano Andrés

Subdirectora General del Libro de la Comunidad de Madrid

Clara Ortega Villanueva

Subdirectora General de Coordinación Bibliotecaria del Ministerio de Cultura y Deporte 


\section{Ricard Pérez Alcázar}

Jefe de Área de Gestión Documental y Administración Electrónica de la Subdirección General de los Archivos Estatales del Ministerio de Cultura y Deporte

\section{Sobre el autor}

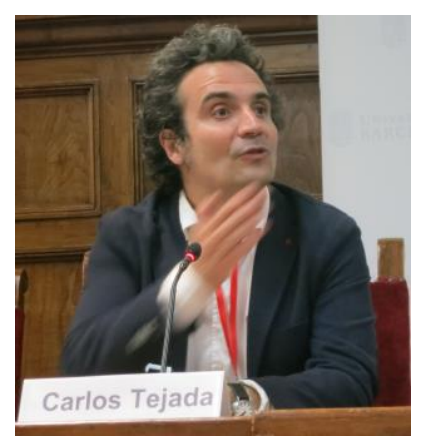

\section{CARLOS MIGUEL TEJADA ARTIGAS}

Profesor Titular de Universidad. Facultad de Ciencias de la Documentación

Es doctor en Documentación y Licenciado en Geografía e Historia. Actualmente es Profesor Titular de la Facultad de Ciencias de la Documentación de la Universidad Complutense de Madrid. Fue coordinador del Grupo de Trabajo de Perfiles Profesionales del Consejo de Cooperación Bibliotecaria del Ministerio de Educación y Cultura de España (2011-2020). Entre sus líneas de investigación figuran los siguientes temas: publicaciones científicas, mercado de trabajo en información y documentación y competencias profesionales. Autor de diferentes publicaciones sobre estas temáticas.

cmtejada@ucm.es 